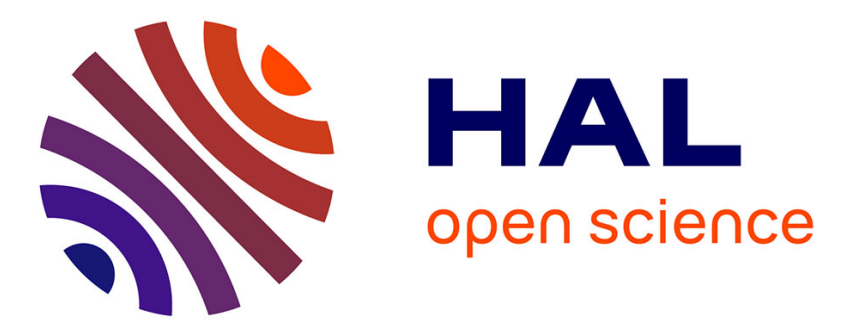

\title{
Resuspension due to vertical oscillations: experiments and numerical modeling
}

Karina Valenzuela-Aracena, Luc Oger, Rodolfo O. Unac, Ana M. Vidales

\section{To cite this version:}

Karina Valenzuela-Aracena, Luc Oger, Rodolfo O. Unac, Ana M. Vidales. Resuspension due to vertical oscillations: experiments and numerical modeling. Granular Matter, 2019, 21 (3), pp.51. 10.1007/s10035-019-0906-6 . hal-02177517

\section{HAL Id: hal-02177517 https://hal-univ-rennes1.archives-ouvertes.fr/hal-02177517}

Submitted on 16 Sep 2019

HAL is a multi-disciplinary open access archive for the deposit and dissemination of scientific research documents, whether they are published or not. The documents may come from teaching and research institutions in France or abroad, or from public or private research centers.
L'archive ouverte pluridisciplinaire HAL, est destinée au dépôt et à la diffusion de documents scientifiques de niveau recherche, publiés ou non, émanant des établissements d'enseignement et de recherche français ou étrangers, des laboratoires publics ou privés. 


\title{
Resuspension due to vertical oscillations: Experiments and numerical modeling
}

\author{
Karina Valenzuela-Aracena • Luc Oger • \\ Rodolfo O. Uñac • Ana M. Vidales
}

Received: March 28, 2019/ Accepted: date

\begin{abstract}
Granular matter is present everywhere in our practical lives. In particular, the movement initiation due to an external excitation, like vibration, is responsible of the detachment of particles that can then be put in an air stream or be part of a pollution process with undesirable consequences. In those scenarios, the knowledge of the mechanisms for the initiation of particle movement is crucial to predict the removal probabilities. This paper focuses on the onset of the movement of particles placed on a horizontal surface subjected to a vertical sinusoidal mechanical vibration. The problem is tracked both experimentally and K. Valenzuela-Aracena · A.M. Vidales · R.O. Uñac

INFAP, CONICET, Departamento de Física, Facultad de Ciencias Físico Matemáticas y Naturales, Universidad Nacional de San Luis, Ejército de los Andes 950, D5700HHW, San Luis, Argentina

E-mail: avidales@unsl.edu.ar E-mail: runiac@unsl.edu.ar

L. Oger

Univ. Rennes, CNRS, IPR (Institut de Physique de Rennes), UMR 6251, F-35000 Rennes, France E-mail: luc.oger@univ-rennes1.fr
\end{abstract}


by DEM simulations. Millimeter glass and plastic spheres with a given size are deposited on a rough surface built with glued glass beads with different packing fractions (coverage). The critical values for the amplitude and the frequency of the vibration needed to start the movement of at least half of the particles on the surface are studied as a function of the properties of the mobile particles and the surface coverage. The results from experiments and simulations are in good agreement. They show that the size of the mobile particle plays the main role in the present problem. Second in relevance are the size ratio between the glued and the mobile particles and the coverage degree of the surface. Finally, the density of the material plays a minor role inside the range of values studied here.

Keywords granular displacement · incipient movement · resuspension · DEM code $\cdot$ vertical oscillation

PACS 83.70.Fn · Granular solids 83.80.Fg · 81.05.Rm · 68.43.Fg

\section{Introduction}

The transport of grains in industrial processes implies, most of the time, energy delivery through agitation in conveyor belts, truck transport, drilling operations, among many others. Phenomena like bed fluidization, the cleaning of surfaces, or the resuspension of particles polluting the atmosphere are based on the existence of a first incipient movement of grains to achieve the detachment and the subsequent dynamic evolution [1-5]. Understanding the mechanisms provoking the destabilization of a deposited particle on a horizontal surface is the key to advance in the description of its dynamic. One of the main perturbations present in many granular matter manipulation stages is the mechanical vibration, either horizon- 
tal or vertical. Naturally, if the vertical acceleration due to vibration breaks the particle mechanical equilibrium, jumps are observed as typical movements for the grains $[6-10]$.

The description of the re-entrainment of a particle when subjected to a vertical vibration implies the analysis of adhesion and capillary forces between the particle and the surface, the inertial force of the particle and its contact interaction with the particles belonging to the surface, especially in cases when rugosity is of the order of (or close to) the size of the beads deposited on it $[11,12]$. As a result, the dynamic of the problem is not straightforward, and the effect of each parameter on the initiation of the particle movement becomes difficult to identify.

In a previous work [13], it has been shown that the critical frequencies needed to destabilize a particle on a vertically vibrated surface decay exponentially with the amplitude of the oscillation and that the frequencies were much lower than those related to the elastic properties of the material constituting the particles. Those experiments were carried on surfaces with a multi-layer of glued spheres, with no control of the extent or distribution of the voids participating in the topography of the surface. In this sense, a deeper study of the critical frequency-amplitude pairs for incipient movement of particles on a highly controlled mono-layer of glued spheres remains an open issue.

To bring some light into the understanding of the problem, a set of experiments is proposed here for spherical particles deposited on a rough surface constructed by a well controlled deposition of grains, glued on it and forming a mono-layer with a given coverage. The surface is then subjected to a mechanical vibration to find which are the critical amplitude-frequency pairs needed to initiate the movement 
of the deposited grains and to determine how are those thresholds modified by the particle size and material and by the surface coverage.

To get a deeper insight, simulations are performed using a Discrete Element Model (DEM) to describe the dynamics of the interactions between the grains and the surface, and to verify the experimental results. As well known, DEM simulations are very helpful to quantify to some extent the role that each of the microscopic interaction parameters has when a granular system is under study $[14,15]$. This insight is not always easy to achieve by direct measurement during experiments.

The experimental set-up and procedure are described in the next section. Then, the section with the experimental results is presented and accompanied by a corresponding discussion. Next, the simulation model is introduced and its results are compared with the experiments. A final discussion is devoted to the whole set of parameters proposed as responsible of the behavior found. Finally, conclusions are presented.

\section{Experimental set up and procedure}

The experimental set up used in the present experiments is sketched in Fig. 1. It consists of a rough rigid surface mounted on a solid plate belonging to a mechanical oscillator (V406). The plate is vibrated thanks to a sinusoidal wave generator (AFG 3011) and an amplifier (PA 100Em) connected to the oscillator. The surface added to the vibrator does not modify the vibration properties of the device given that the added mass is small compared to that of the vibrator. In this way, both frequency and amplitude of the oscillatory movement can be controlled properly. 


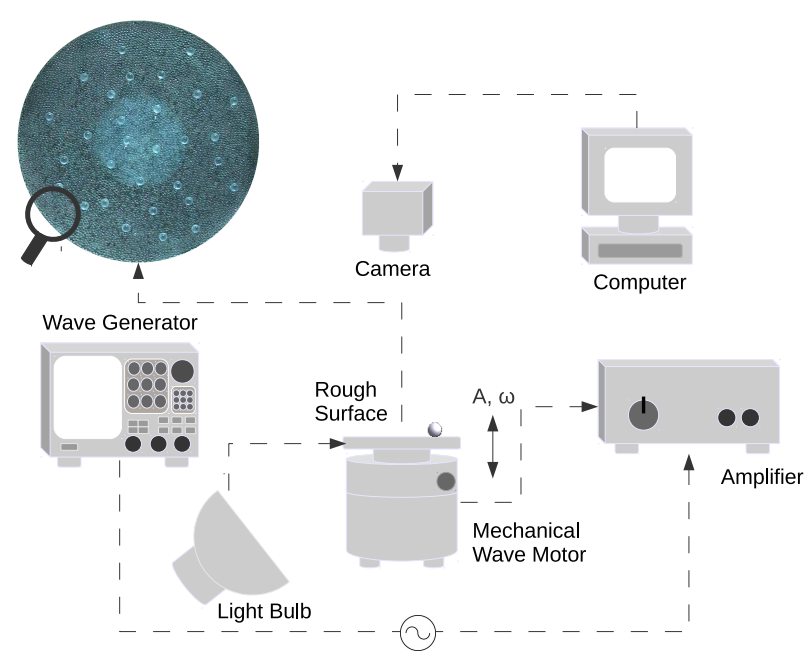

Fig. 1 Sketch of the experimental set-up used in all the experiments. The different elements are indicated.

A video camera is mounted to film the whole rough surface and the mobile beads with an appropriate illumination. The camera is connected to a $\mathrm{PC}$ for data record and processing.

The rough surfaces are built by gluing spherical mono-sized glass beads to a glass disk (plate) whose diameter is $18 \mathrm{~cm}$, using an EPOXI type adhesive (Araldite 2011). The beads are randomly poured on the surface in such a way that only a mono-layer of particles is created with a quite homogeneous spatial distribution. This mono-layer allows controlling the extent of the three contacts (triplets) holding a moving sphere.

The number of grains glued on the surface is calculated such that the final coverage of the plate, $\theta$, is known. Four different values of $\theta$ are tried, i.e., $50 \%$, $66 \%, 70 \%$ and $82 \%$. In particular, the construction of the rough surfaces is not always easy at low coverage because the mobile beads may be in contact with the glued surface for the particles' sticking. For that reason, we only work with 
coverages that prevent the underlying plane surface of being touched by the moving grains.

A given percentage of coverage is calculated as the ratio between the projected area of the glued beads on the horizontal plate surface divided by the plate surface and multiplied by 100. Two different sizes are chosen for the glued grains, $1 \mathrm{~mm}$ and $2 \mathrm{~mm}$, separately. Thus, two type of surfaces are created, i.e., $S 1$ and $S 2$, respectively. As a result, eight different rough surfaces are generated using this procedure.

The mobile spheres to be deposited on the surface for vibration are of two different materials, glass and ABS (Acrylonitrile Butadiene Styrene). They have different diameters, $D$, equal to $3 \mathrm{~mm}, 6 \mathrm{~mm}, 7 \mathrm{~mm}$ and $10 \mathrm{~mm}$ for glass particles and $3 \mathrm{~mm}$ and $5 \mathrm{~mm}$, for ABS, all with a size dispersion of $1 \%$.

In order to have a good control of the input amplitude, $A$, for the vibrational excitation of the surface, we calibrate the corresponding amplitude of the oscillator arm (responsible of the surface movement) with the help of a video camera and image analysis. A linear correspondence is found between the amplitude and the input voltage. In this way, ten different amplitudes are calibrated and used in the experiments, i.e., $1.5,1.9,2.2,2.6,3.0,3.4,3.7,4.1,4.5,4.8 \mathrm{~mm}$, with a negligible error.

A typical experimental run starts by the random deposition of 30 grains of a given material and size on the rough surface. Once the particles are placed, the video camera is turned on and the surface is subjected to a sinusoidal vibration at a given $A$, fixed for the rest of that run. The frequency is changed following a continuous ramp at a rate of $1 \mathrm{~Hz}$ every 6 seconds until all the grains initiate their movement. At that time, the vibration stops and the video is finished and 
saved. Then, a new set of grains is distributed on the surface and a new run, with a different amplitude, is started. The procedure is repeated until the ten different values for $A$ are tested. As for the case of calibration, all the runs are recorded with a Full HD camera with 60 frames/second. As a result, a total of 10 runs (one for each $A$ value) for each surface ( $S 1$ and $S 2), \theta, D$ and material (glass or ABS) is obtained.

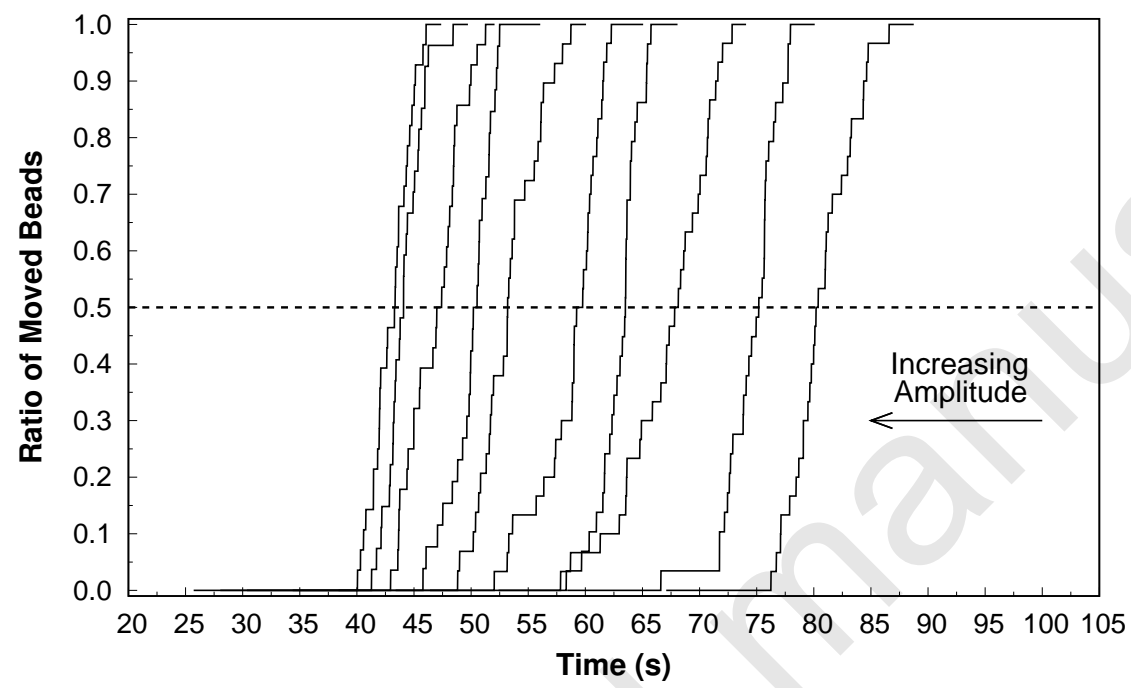

Fig. 2 Example of the cumulative counting rate of moved particles vs. time for $\theta=70 \%, S 1$, $D=6 \mathrm{~mm}$ glass spheres and all amplitudes. The horizontal line cuts the curves to determine the critical time (thus, the frequency) at which half of them are in movement.

For a given run, the critical frequency, $w_{c}$, is defined as the one at which the grains are vertically vibrated when, at least, the half of them has horizontally moved. To determine the critical frequencies, each recorded video is analyzed with the help of an open source image processing program like ImageJ. With this processor, we perform an optimized track of each particle on the surface. A typical cumulative counting of the moving particles as a function of time is shown in 
Fig. 2 for the case of glass beads with $6 \mathrm{~mm}$ on the $S 1$ surface with $\theta=70 \%$ and for all the amplitudes studied. The horizontal line at ratio 0.5 of moving beads intercepts the curves determining the corresponding critical frequencies (linked to the corresponding time values) for each amplitude. As indicated, $A$ increases from right to left.

\section{Experimental results}
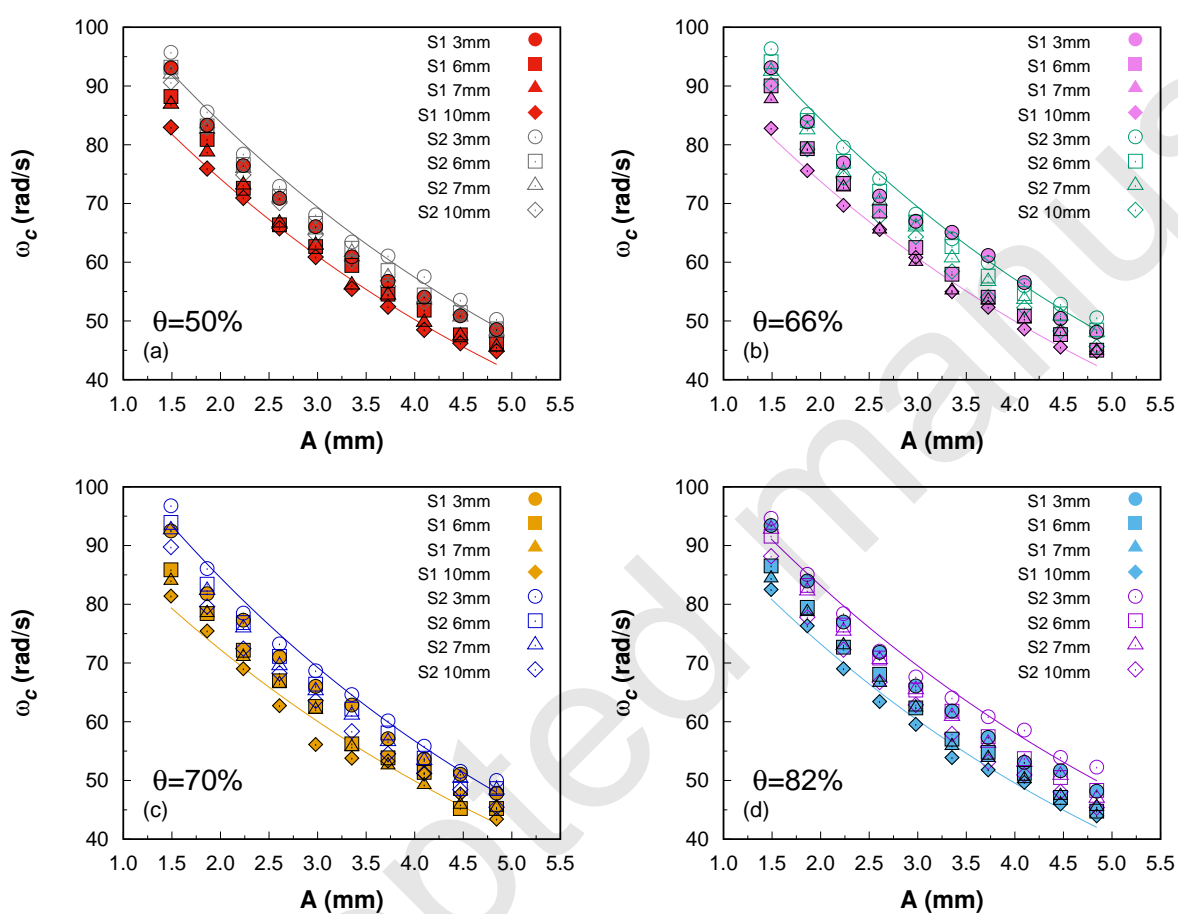

Fig. 3 Critical frequencies as a function of the amplitude of the vibration for glass spheres on surfaces $S 1$ and $S 2$. The coverage is indicated in each part of the figure. Filled and open symbols correspond to surfaces $S 1$ and $S 2$, respectively.

The curves shown in Fig. 3 result from plotting the critical frequencies, $\omega_{c}$, obtained by following the procedure depicted above. The data belong to the case 
of glass spheres on both surfaces, $S 1$ and $S 2$. Each figure corresponds to a different coverage, from $50 \%$ to $82 \%$. The sizes of the particles are indicated along with the corresponding surface used in each experiment.

The decaying behavior is evident. Thus, in order to evaluate in more detail the dependence of this behavior on the size of the particles, on the characteristics of the surface and on the eventual change of the material of the moving spheres, the following fitting function is assumed for each set of data:

$$
\omega_{c}(A)=\omega_{c}(0) \exp (-\gamma A)
$$

where $\gamma$ accounts for the extent of decay and $\omega_{c}(0)$ is the finite frequency value expected as the amplitude tends to zero in a real damped system. Each of the experimental data sets in Fig. 3 can be fitted with Eq. 1. To avoid the superposition of curves, only the exponential fits performed on the extreme cases are shown. The critical frequencies for surface $S 2$ are systematically greater than for $S 1$, related to the effect of the greater size of the holes (or voids) formed by the beads glued to $S 2$.

The exponential behavior found here is in agreement with the one reported in [13] and the range of values for the frequencies is again quite lower than that derived from the elastic properties of the materials involved $[1,2]$.

By fitting the experimental data with Eq. 1 (leaving as free parameters both $\gamma$ and $\left.\omega_{c}(0)\right)$ a set of values that can be correlated with the experimental parameters is obtained, as seen in Fig. 4. There, one can see a certain monotonic dependence of $\omega_{c}(0)$ on the particle diameter $D$ for the case of glass spheres and each $\theta$. Indeed, although the relative variation is less than $10 \%$, it is evident that, as the size of 


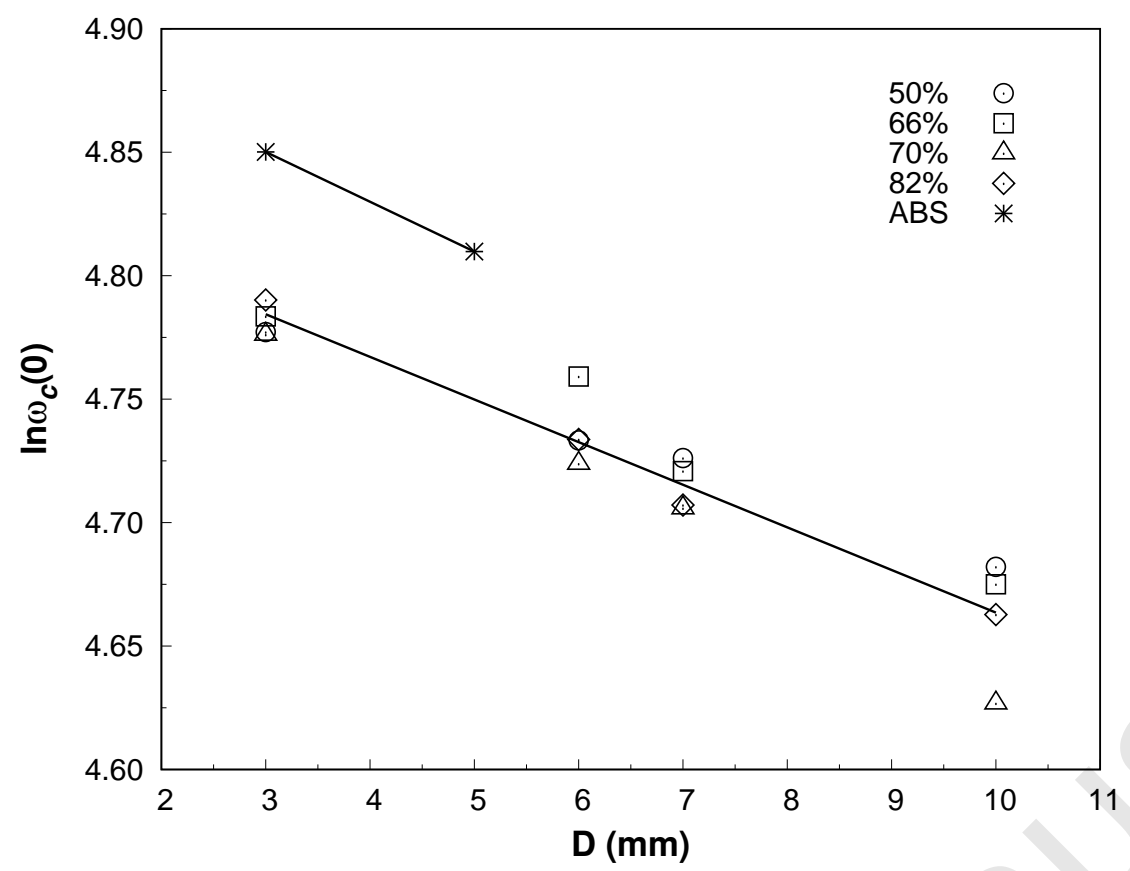

Fig. 4 Correlation between $\ln w_{c}(0)$ and the particle diameter for $S 1$. The ABS values are an average over all coverages.

the particle is greater, the critical frequency for $A \rightarrow 0$ is lower. This trend is in agreement with the general behavior of our results, where, for a given amplitude, the larger the particles the lower the frequencies for destabilization. The data corresponding to ABS is discussed below and is presented as an average value over the four values of $\theta$.

On the other hand, Fig. 5 shows that the decaying constants for all the exponential curves fluctuate around a value of 0.19 , being practically the same independently of the particle diameters. In both figures, the influence of the coverage is not decisive, at least for the percentages evaluated in the present work. In this figure again, the data for ABS is presented as an average over the coverage and is discussed further below. 


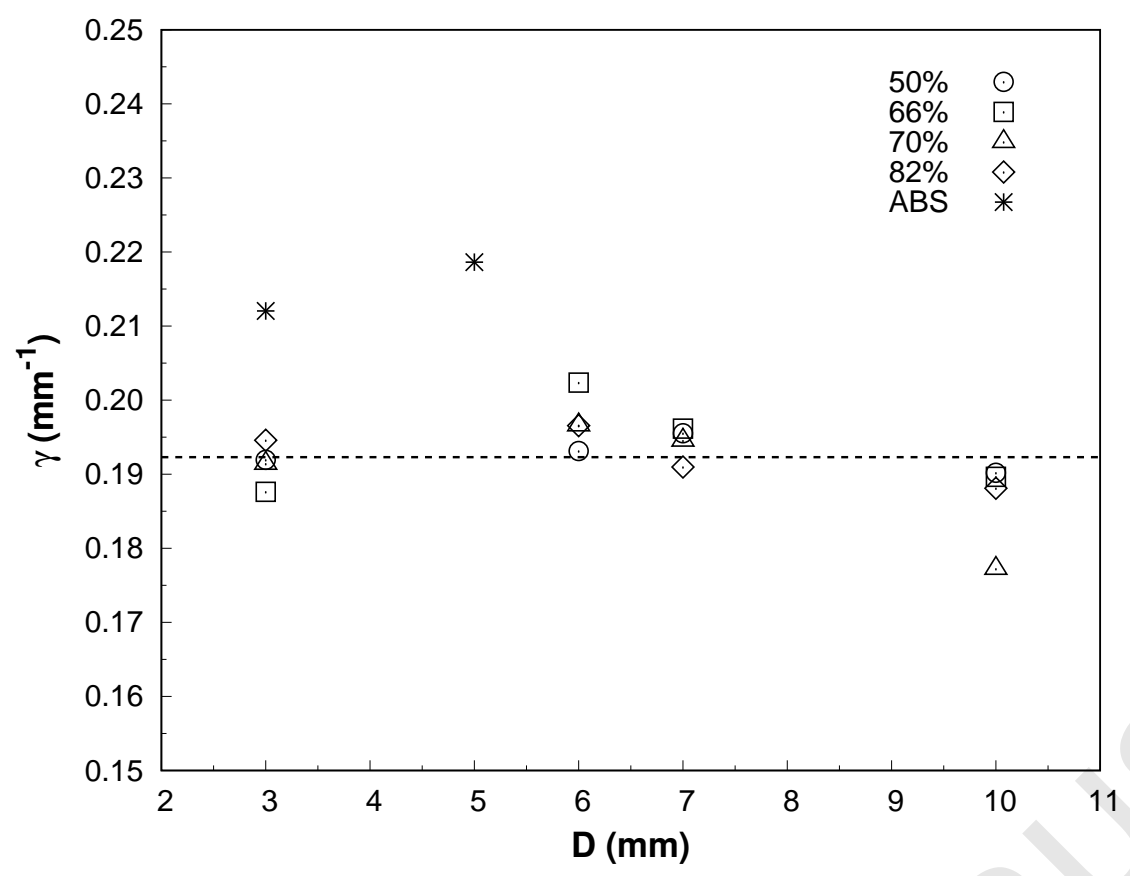

Fig. 5 Correlation between the exponent $\gamma$ (Eq.(1)) and the particle diameter for $S 1$. The ABS values are an average over all coverages.

In Fig. 6 and Fig. 7 we show the results for $\omega_{c}(0)$ and $\gamma$ for the moving particles on surface $S 2$. Again, a decreasing trend is observed for $\omega_{c}(0)$ with the particle diameter and a nearly constant value for $\gamma$, independently of the size. For this surface, there is a difference in the behavior at coverage $82 \%$. This effect could be due to local inhomogeneity generated during the construction of the surface, as seen below. In both figures, the corresponding values for ABS are presented, also averaged over the coverage.

To get an idea of the effect of the material on the critical frequencies for destabilization, we perform a set of experiments depositing spheres of ABS with $3 \mathrm{~mm}$ and $5 \mathrm{~mm}$ diameters. Given that only two different sphere sizes are used and that $\theta$ has a small effect, the average of the values is taken for the four coverages 


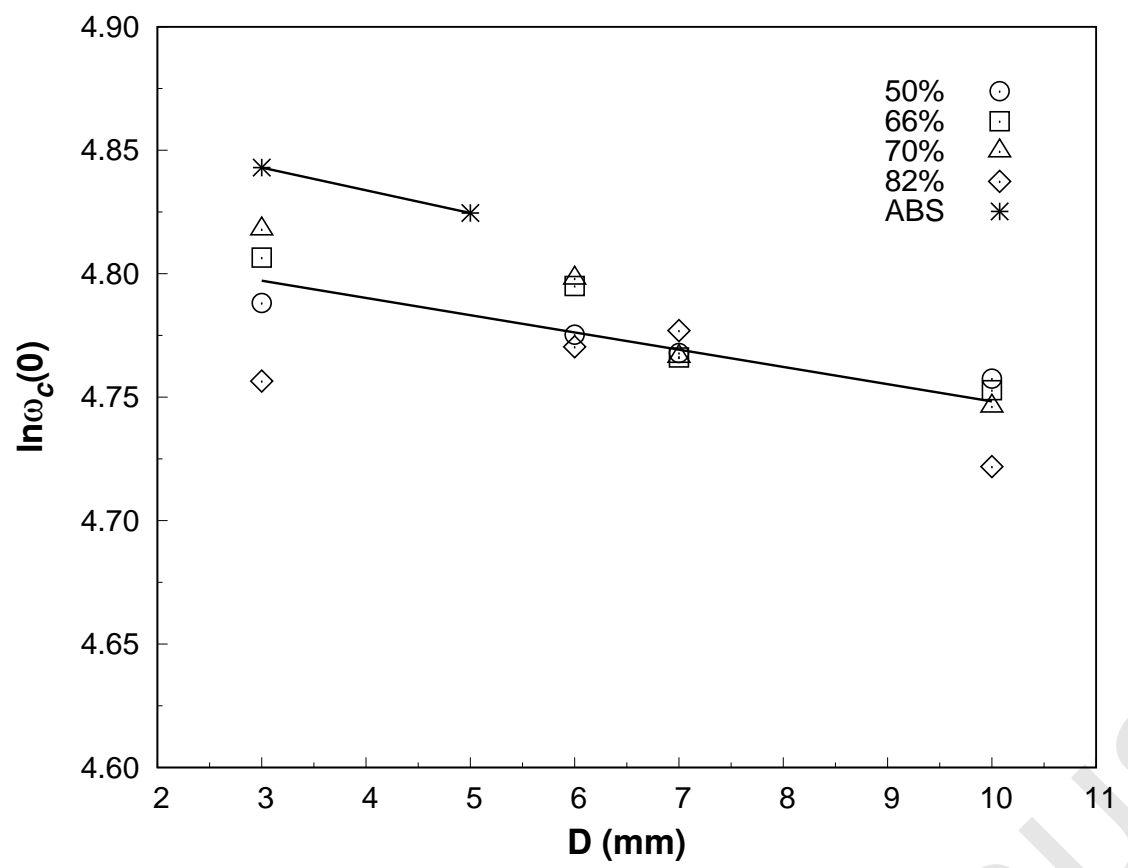

Fig. 6 Correlation between $\ln w_{c}(0)$ and the particle diameter for $S 2$. The ABS values are an average over all coverages.

and represented in Fig. 4 and Fig. 5 by asterisks. In both surfaces, the values for $\omega_{c}(0)$ for ABS fall above those for glass. The decaying constants show to depend slightly on the material of the particles, i.e., $\gamma$ for ABS is higher than for glass. This means that the critical frequencies are sensitive to the material of the beads, but only weakly, because the difference observed in the figures is less than a $3 \%$ for the case of $\omega$, and $10 \%$ for the case of $\gamma$. It is interesting to note that the dependence of $\omega_{c}(0)$ on the size of the particles seems to be the same for both materials, given that the lines in Fig. 4 and Fig. 6 have the same slopes. We will also analyze the effect of the material later in the simulation section.

The little effect of $\theta$ on the previous results could be explained by looking at the distribution of voids on the surface as a function of the size of the particle deposited 


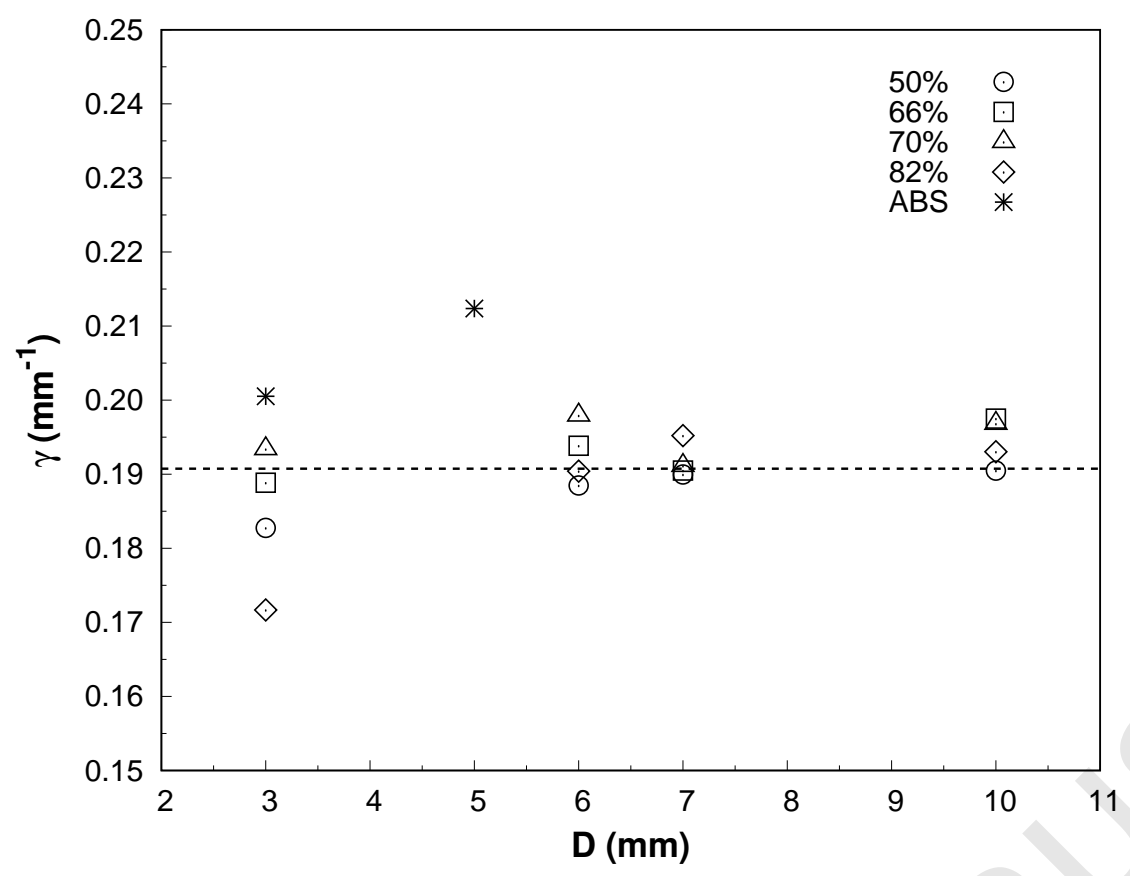

Fig. 7 Correlation between the exponent $\gamma$ (Eq. 1) and the particle diameter for $S 2$. The ABS values are an average over all coverage values.

on that surface. To accomplish this, a disordered rough surface is created using a simulation of a random 3D deposit of spheres. The procedure will be explained below in Section 4. For example, for the case of a $3 \mathrm{~mm}$ particle on $S 2$, which is the most unfavorable case, most of the voids are expected to be smaller than the size of that particle. One can check this affirmation by looking at Fig. 8, where the cumulative probability of finding a void with its size scaled by the particle diameter is calculated on the simulated surface. For all coverage values, around $90 \%$ of the voids have a size smaller than the diameter of the particle. Only for the case of $50 \%$ coverage, one finds a $10 \%$ probability of a void size greater than the particle size. In this way, it could be said that, for the experimental cases studied here, the effect of the coverage is expected to be weak. 


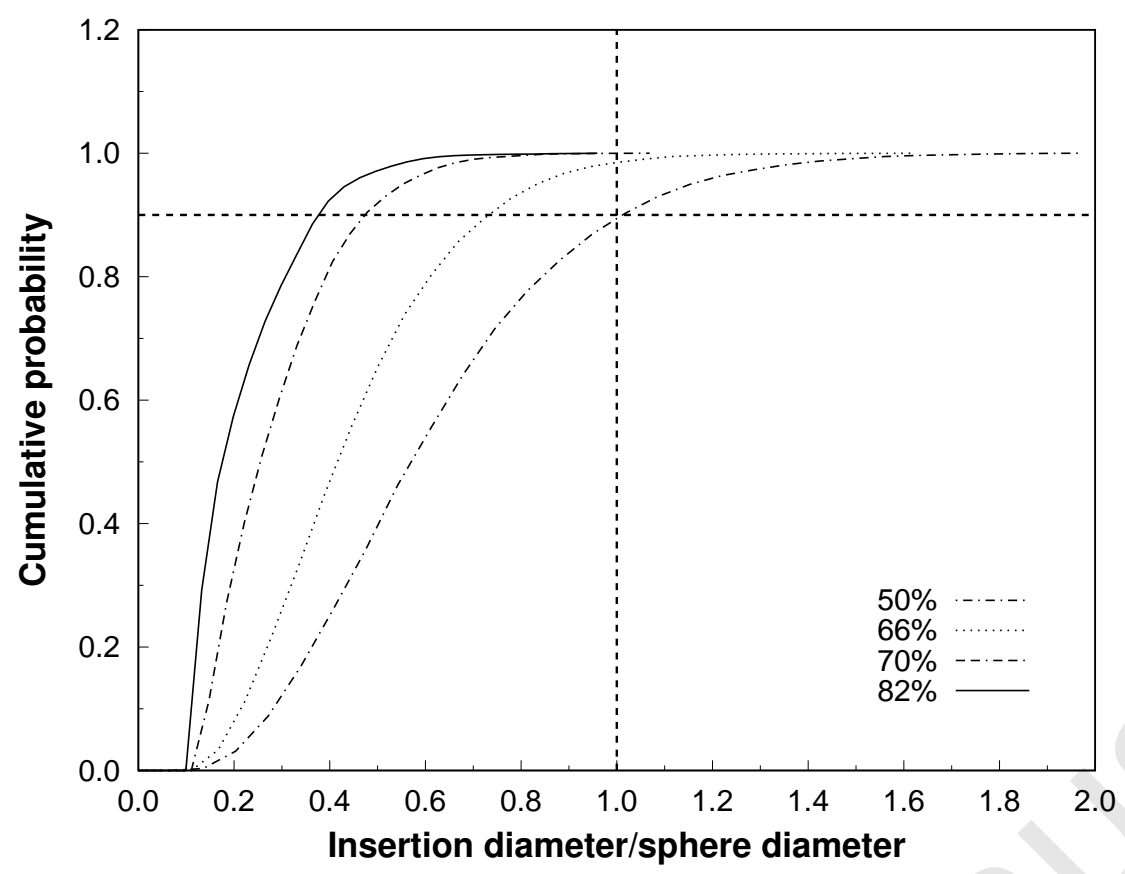

Fig. 8 Cumulative probability of finding a void having a certain diameter scaled by the moving particle diameter. This probability is calculated with the help of a Voronoi tessellation analysis to be depicted in the numerical simulation section below.

3.1 Qualitative theoretical approach

The qualitative model outlined in this section attempts to demonstrate that the experimental behavior found above is, at least, expectable from a simple geometrical point of view.

The sketch in Fig. 9 provides a possible theoretical view of what one would expect as a critical threshold for destabilization of the moving grains. The torque needed to start the rolling of the particle and thus to overcome the underlying particle belonging to the surface, without considering friction, simply leads to $a_{c}=g \tan \theta$, where $\theta$ is such that $\sin \theta \approx r /(r+R), r$ being the radius of the 
glued beads and $R=D / 2$, the radius of the moving sphere. This means that the acceleration is expected to inversely decrease with the size of the spheres.

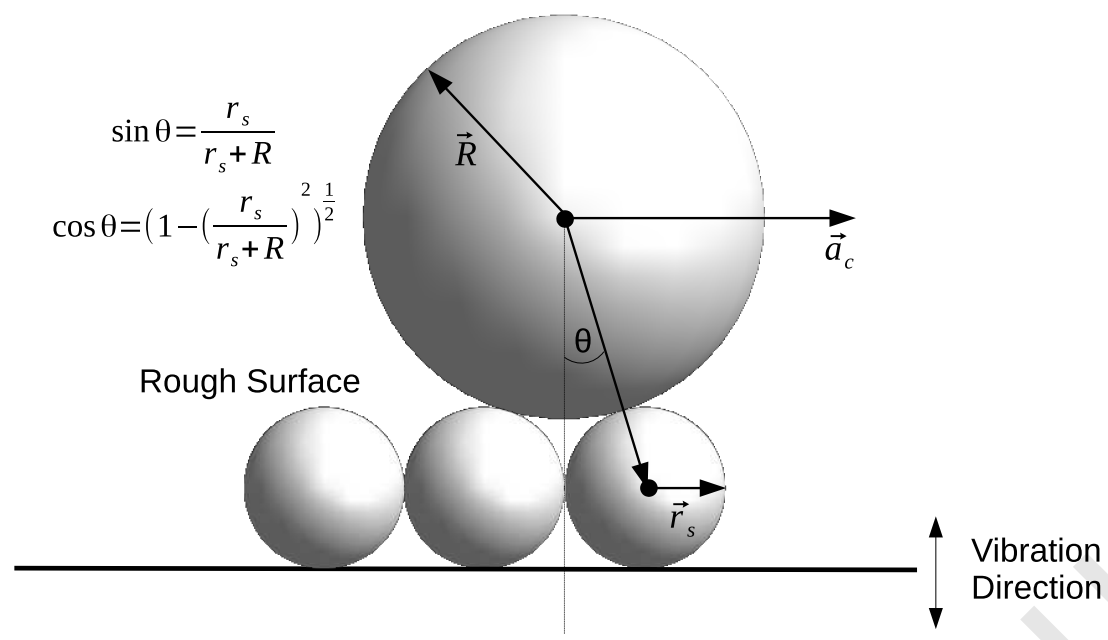

Fig. 9 Sketch of a moving sphere on top of two beads belonging to the surface and submitted to a destabilizing acceleration $a_{c}$.

To verify the above expectation, we can calculate the quantity $w_{c}^{2} A$, which is the acceleration applied to the particle by the vibrating surface at the moment of destabilization. To relate this acceleration with the one needed to actually destabilize the sphere, one has to keep in mind the picture of the sphere deposited on top of three surface beads. The contacts with the three beads (forming a "triplet") do not have to be at the same height respect to the horizontal. The destabilization force will be the resultant of the forces applied on the particle by the three contacts, which, in turn, are proportional to the force applied by the plate. In an equilibrium scenario, these forces cancel out but, any local perturbation setting an asymmetry in the contacts will produce a resultant different from zero and proportional to $w_{c}^{2} A$. 
Calculating this acceleration for all the amplitudes and critical frequencies obtained in the experiments and averaging for each sphere size and material, the plots presented in Fig. 10 are obtained. The acceleration effectively decreases with the size of the spheres. Here again, the effect of the coverage degree is slight, for that reason, data is averaged over all $\theta$. The values for ABS fall in the same range than those for glass. This is in agreement with the fact that $a_{c}$ does not depend on the material. The accelerations for $S 2$ are systematically greater than for $S 1$. This is understood because the sizes of the voids for $S 2$ are greater than those for $S 1$ and, consequently, the heights for the triplet of contacts are more likely to be different than for $S 1$.

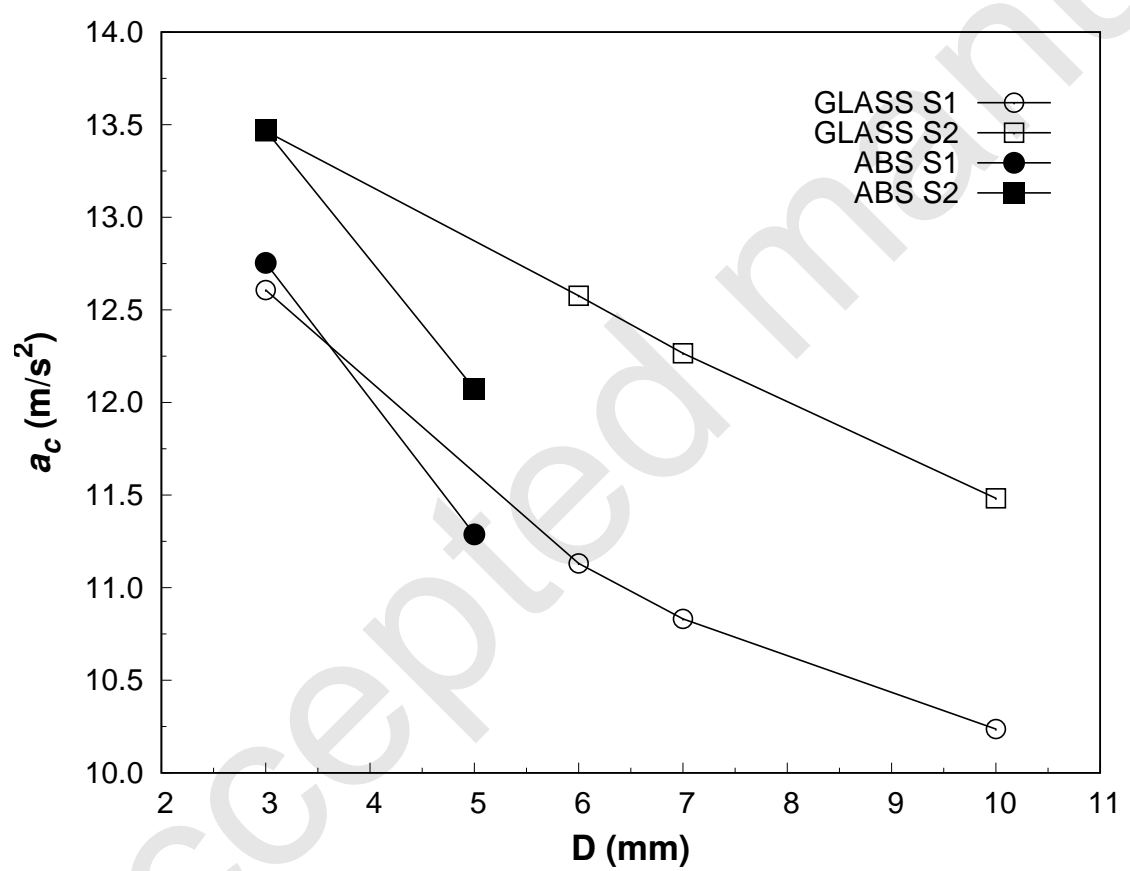

Fig. 10 Acceleration $a_{c}=w_{c}^{2} A$ vs. the particle diameter for $S 1$. The values are the result of an average over the four values of the coverage $\theta$. 


\section{Numerical simulations}

4.1 Particle contact model: Spring-dashpot model

Numerical simulations are not only useful to validate experimental behavior, but especially helpful to inspect parameter ranges that are difficult to achieve at the laboratory. In the present case, effects of particle density, particle size and surface coverage may be identified in an easier way through the use of a classical DEM molecular dynamics model. As explained before, low coverage is not tried in the present experiments. In this way, simulations become useful to investigate the effect of lower values of $\theta$. Besides, the low ratio limit (small moving particles) cannot be reached in the experiments due to the difficulty for detecting the movement of particles when their size is close to that of the glued spheres on the surface.

The present simulation model is also known as "soft spheres", because the particles can overlap in addition to slipping and rotation. It follows the twodimensional formulation of Savage [14] and the three-dimensional extension used by Oger et al. [15]. The spherical particles can have multiple contacts that can persist for extended duration (typically during 50 time steps). In the present case, those contacts can arise at the initial stage of the packing formation or during the oscillation process. Both normal and tangential forces develop at the contact between two spheres.

As it is common in most simulation codes, one can access at any time to all the data for each ith particle, i.e., its radius $R_{i}$, the position of its center of mass $\left(x_{i}, y_{i}, z_{i}\right)$, its velocity, acceleration and to its polar angle $\theta_{i}$ and azimuth angle $\phi_{i}$.

When two spheres overlap, the normal and tangential contact forces increase as the centers of the particles approach each other. The normal force $F_{n}$ at the contact 
is modeled as viscoelastic. It consists of an elastic (linear spring) contribution and a viscous damping (linear dashpot) contribution, described as follows:

$$
\begin{array}{ll}
\text { compression }: & F_{n}=K_{n} \delta-b_{n} v_{n} \\
& \text { for } \delta=\left(\sigma-\left|\mathbf{r}_{\mathbf{i}}-\mathbf{r}_{\mathbf{j}}\right|\right)>\mathbf{0} \\
\text { tension : } & F_{n}=0 \\
& \text { for } \delta<0
\end{array}
$$

where $K_{n}$ is the spring constant for normal forces, $\delta$ is the relative normal displacement between the centers of the two particles in contact, $\sigma$ is the distance vector between the two centers, $\mathbf{r}_{\mathbf{i}}$ and $\mathbf{r}_{\mathbf{j}}$ are the two radii of the particles, $v_{n}$ is the relative normal velocity and $b_{n}$ is the dashpot constant for normal forces.

The force in the tangential direction is also modeled as a viscoelastic one; a linear spring and a linear dashpot are used to generate a tangential contact force as follows:

$$
F_{t}=K_{t} \delta_{t}-b_{t} v_{t}
$$

where $K_{t}$ is the spring constant for tangential forces, $\delta_{t}$ is the relative lateral displacement during all the duration of the contact, $v_{t}$ is the relative tangential velocity, and $b_{t}$ is the dashpot constant for tangential forces. The tangential force, $F_{t}$, is also limited to a maximum value which is chosen according to a Coulomb friction law when slipping can occur, i.e.:

$$
F_{t}=\mu F_{n}
$$

where $\mu$ is the friction coefficient. The tangential force acts in a direction opposite to that of the relative tangential velocity $v_{t}$.

The presence of capillary forces between the particles is also considered through the development of tensile normal and tangential forces at the contacts. A tensile 
(negative) value is possible up to a limiting tensile strength defined for the bond (i.e., breaking of a wetting film). If the normal (or the tangential) tensile force exceeds that limit, the bond breaks and the normal (tangential) force follows the standard rule for positive values. In order to introduce this cohesive term in a simple manner, we have followed the results of the work by Crassous and Charlaix [16] which show a linear evolution of the cohesive force during the pulling out of two spheres. Depending of the humidity rate, the amount of liquid surrounding each sphere can be calculated, delimiting thus the distance between spheres where the cohesive force remains constant and equal to the capillary force. The calculation of the force is obtained with the liquid surface tension, $\gamma_{l v}$, the contact angle on the solid surface, $\theta$, and the radius of curvature, $R$ in this case, as follows:

$$
F_{c a p}=e \pi \gamma_{l v} R \cos \theta
$$

with $e=2$ in the limit of rigid surfaces and $e=3 / 2$ in the limit of soft elastic surfaces. In this way, when the two spheres pull apart each other, the force decreases linearly as a function of the distance and the liquid amount thickness. The slope of this linear behavior is defined by Crassous et al. [17] in Eq. (9) of that paper.

Finally, in order to simulate non perfectly spherical particles, a new numerical term defined as rolling resistance coefficient has been introduced recently [1820]. This coefficient is defined as a nondimensional term related of the particle eccentricity and takes into account the effect of the shape of the particle on its ease for rolling. The rolling resistance is denoted by:

$$
\vec{q}^{\text {rolling }}=-\mu_{r} R_{i} \omega_{i}\left|\overrightarrow{F_{i j}^{n}}\right|
$$

where $\mu_{r}$ is the rolling friction coefficient (dimensionless), $R_{i}$ is the particle ra-

dius, $\overrightarrow{F_{i j}}$ represents the normal force (including elastic and damping) imposed on 
particle $i$ by particle $j$, and $\omega_{i}$ is the unit vector for the angular velocity of particle $i$. This rolling resistance is added to the torque component in order to decrease the rolling velocity. Finally, no long range interactions are present in this model.

4.2 Nondimensional approach

It is convenient to cast the governing Eqs. (2) and (3) in nondimensional form and perform the computations based upon these dimensionless equations (Savage, 1992). In using the nondimensional form of the equations, we have the advantage of being able to easily reach results for different physical scales as, for instance, from fine powders of $10 \mu \mathrm{m}$ up to ice floes of $100 \mathrm{~km}$, provided the appropriate effective spring constant and the time scale for one collision are used. Besides, it is straightforward to revert back to physical variables when needed.

Hence, all variables are nondimensionalized. For example, lengths are divided by $D$, the diameter of the largest particle used in the computations; time is divided by $\sqrt{M / K_{n}}$, where $M$ is the mass of the largest particle and $K_{n}$ the effective spring constant used in Eq. (2) and velocities are divided by $D / \sqrt{M / K_{n}}$. In this way, nondimensional time and spatial coordinates become:

$$
\begin{gathered}
(\tilde{t}, \tilde{x}, \tilde{y}, \tilde{z})=\left(t \sqrt{\frac{K_{n}}{M}}, \frac{x}{D}, \frac{y}{D}, \frac{z}{D}\right) . \\
\left(\tilde{v}_{x}, \tilde{v}_{y}, \tilde{v}_{z}, \tilde{\omega}, \tilde{\Omega}\right)=\sqrt{\frac{M}{K_{n}}}\left(\frac{v_{x}}{D}, \frac{v_{y}}{D}, \frac{v_{z}}{D}, \omega, \Omega\right) .
\end{gathered}
$$


By using Eqs. (2) and (3) with the terms of the nondimensional variables (7) and (8) we obtain:

$$
\begin{gathered}
\frac{d \tilde{v}_{x}}{d \tilde{t}}=\sum_{i=1}^{n_{c}^{i}} \frac{\tilde{k}}{4 \tilde{r}^{2}}\left(\tilde{\delta}_{i}\right)_{x} \\
\frac{d \tilde{v}_{y}}{d \tilde{t}}=\sum_{i=1}^{n_{c}^{i}} \frac{\tilde{k}}{4 \tilde{r}^{2}}\left(\tilde{\delta}_{i}\right)_{y} \\
\frac{d \tilde{v}_{z}}{d \tilde{t}}=\sum_{i=1}^{n_{c}^{i}} \frac{\tilde{k}}{4 \tilde{r}^{2}}\left(\tilde{\delta}_{i}\right)_{z} \\
\frac{d \tilde{\omega}}{d \tilde{t}}=\sum_{i=1}^{n_{c}^{i}}\left(\operatorname{sign}_{i}\right) \mu_{i} \frac{\tilde{k} \tilde{\delta}_{i}}{2 \tilde{r}^{3}} \\
\frac{d \tilde{\Omega}}{d \tilde{t}}=\sum_{i=1}^{n_{c}^{i}}\left(\operatorname{sign}_{i}\right) \mu_{i} \frac{\tilde{k} \tilde{\delta}_{i}}{2 \tilde{r}^{3}}
\end{gathered}
$$

where $\tilde{\delta}=\delta / D$ and $\tilde{r}=r / D$ are the dimensionless distance and spring constant $\tilde{k}=k / K_{n}$. The term $\operatorname{sign}_{i}$ specifies that the direction of the $i$ th tangential contact force acting on this particle is in the opposite direction of the relative shear velocity acting on the two particles.

\subsection{Initial preparation}

In order to simulate the experimental set-up presented in the previous section, the numerical code creates for the first time the rough surface of glued spheres, i.e., immobile centers in the referential moving plate. Then, the "moving" spheres of a given size and material properties are deposited on the rough surface for their future motion tracking. Finally, all the system is put under oscillation as in the experiments.

According to the experimental conditions, the best way to simulate the disordered rough surface is to create a random $3 \mathrm{D}$ deposit of spheres. At the end of this 
deposition process, all the spheres are sitting on a stable position under gravity with one contact with the solid plate. Thereafter, all the spheres are assumed as fixed in their positions and they only interact with the upper moving spheres. At the end, a number between 4500 (35\% of coverage) and up to 10500 (82\% of coverage) of fixed particles is reached.

In this way, a disordered 3D surface is built. In order to analyze the local and global composition of the surface, a Voronoi tessellation is applied (Fig. 11). Indeed, for a small packing fraction $(\approx 30-50 \%)$, few beads are in contact, for that reason, neighbors have to be defined without any ambiguity.

By doing this unique tessellated pavement of the surface, all the local arrangements formed by three neighboring beads can be determined, where one can place a moving sphere that can geometrically fit on the hole defined by that triplet of glued particles. The distribution of holes for different coverages is shown in Fig. 12.

Once the rough surface is created, moving spheres are randomly deposited on stable positions all over the surface in order to obtain a large number of different situations spanning all possible stable conditions. During this step, the spheres are not allowed to overlap with the rough surface, neither to touch other moving spheres.

\subsection{Plate oscillation}

After the above initial steps, where only geometrical considerations are taken into account, the simulation of the dynamics starts by adding the gravitational field, the cohesive term (capillary forces) and all the mechanical parameters, like restitution, friction and rolling resistance coefficients. In this way, the mechanical interaction 


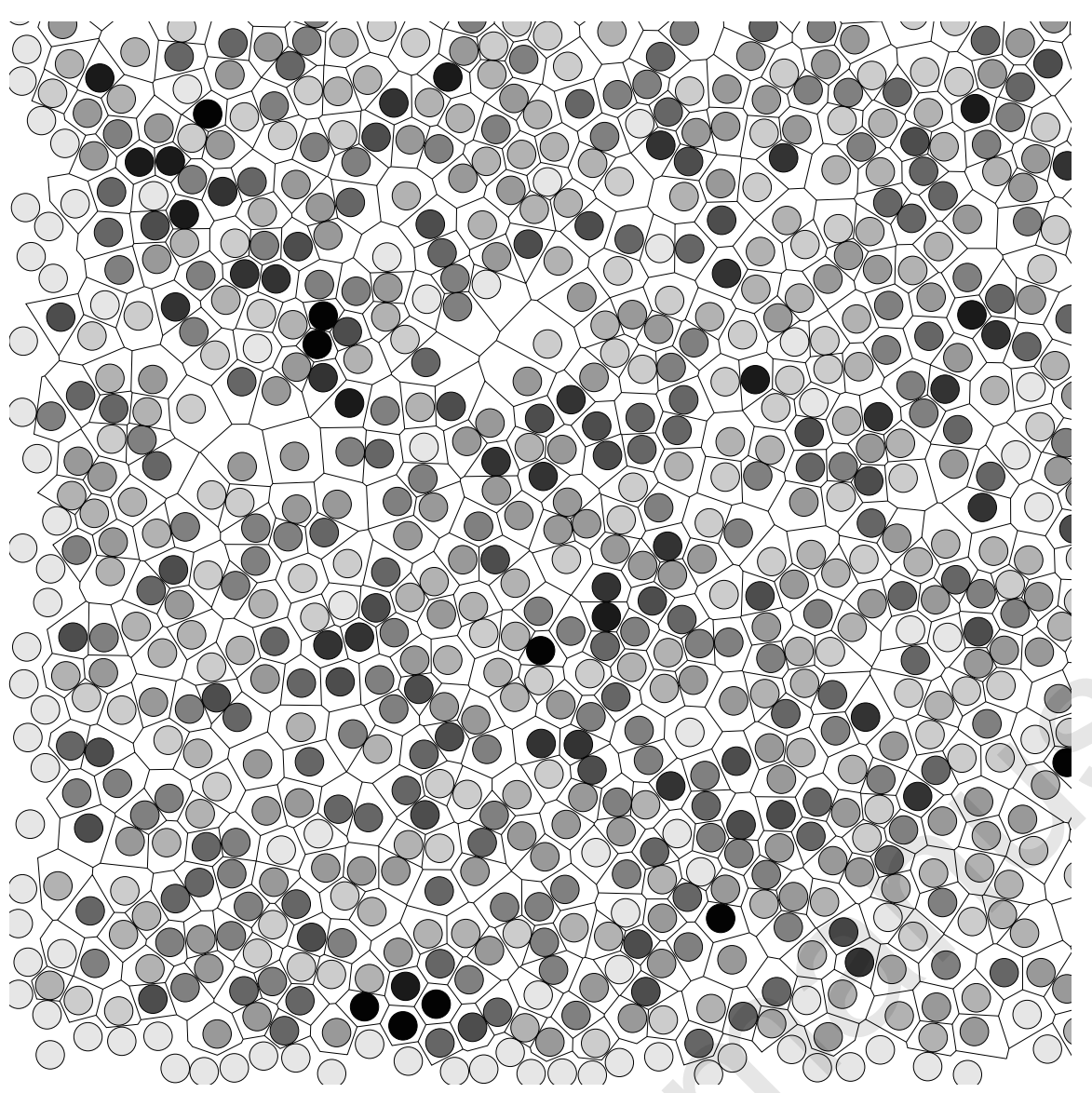

Fig. 11 Description of a rough surface with $\theta=40 \%$ with the 2D Voronoi tessellation for spatial calculation. The color is linked to the definition of the local order $\phi_{6}$ introduced by Torquato [21]. Higher gray means higher local order.

between moving particles and the surface begins. This step is run during a time long enough to produce the overlapping process of the upper spheres with the glued ones, and also to provoke the apparition of the cohesive forces between spheres.

The vibration of the sample is achieved by performing a vertical oscillation of the glued spheres constituting the plate with a given amplitude and frequency. Due to the interactions, this process will automatically generate the oscillation of all the spheres. It is worthy to mention here that this oscillation will limit the choice 


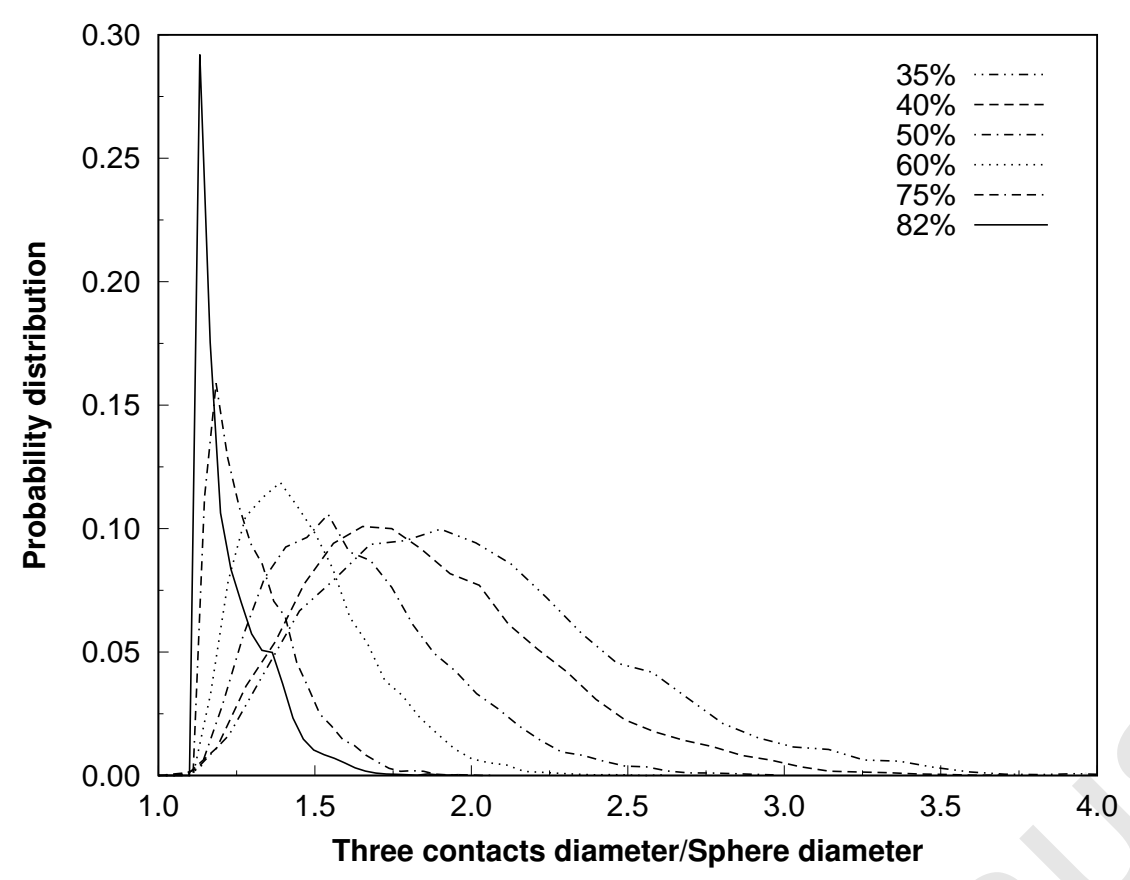

Fig. 12 Distribution of the circle diameter passing through the centers of three neighboring spheres. The coverage of the $2 \mathrm{D}$ surface is varying from $35 \%$ up to the maximum dense disorder one $82 \%)$

of the time step for simulations. Indeed, besides the classical 1/50 duration time for collisions, it is necessary to take into account that the displacement provoking the oscillation of the glued beads will generate an overlap with the upper spheres that has also to follow the soft DEM rules. The positions of the moving spheres are always linked to the plate coordinates in the relative moving frame.

In contrast to the experimental procedure, where the oscillation frequency is continuously increased by successive steps, we adopt another approach for searching the frequency-amplitude threshold. Indeed, increasing the frequency step by step would consume a very long computer time, as it would be necessary to wait long enough at each small frequency increment in order to evaluate whether at 
least half of the beads have moved or not. Fortunately, in our code, it is possible to manage a large number of moving spheres if one prevents the particles to see each other (no billiard balls effects). Thus, by running a few times for each frequency-amplitude pair, and looking for spheres in movement after a given time, the $50 \%$ of moving spheres threshold can easily be reached.

\section{Numerical results}

5.1 Choice of the simulation parameters

In order to optimize the running time and to have a representative number of moving particles for good statistics, the dependence of the results on the initial number of deposited spheres is explored. Fig. 13 shows two examples of the evolution of the ratio of moved spheres as a function of their initial number on the surface. When the size ratio between the deposited and the glued particles is 2 , a number of 100 initial particles is at least needed for the results to be independent of the initial number. When the size ratio is 3 , the minimum number is 200. Following this observation, the maximum between 1000 and the $6 \%$ of the total number of glued beads on the surface is chosen as the number of deposited spheres. Because the moving particles do not see each other, their number is also quite enough for exploring all the possible geometrical configurations of tripods supporting a sphere.

Simulations are also run to determine the best values for the parameters intervening in the force models and in order to represent the experimental evidence of the decaying behavior of $\omega_{c}$ with the amplitude. As a conclusion, a slight dependence on stiffness values going from $10^{4} k N$ to $10^{6} k N$ and for rolling resistance 


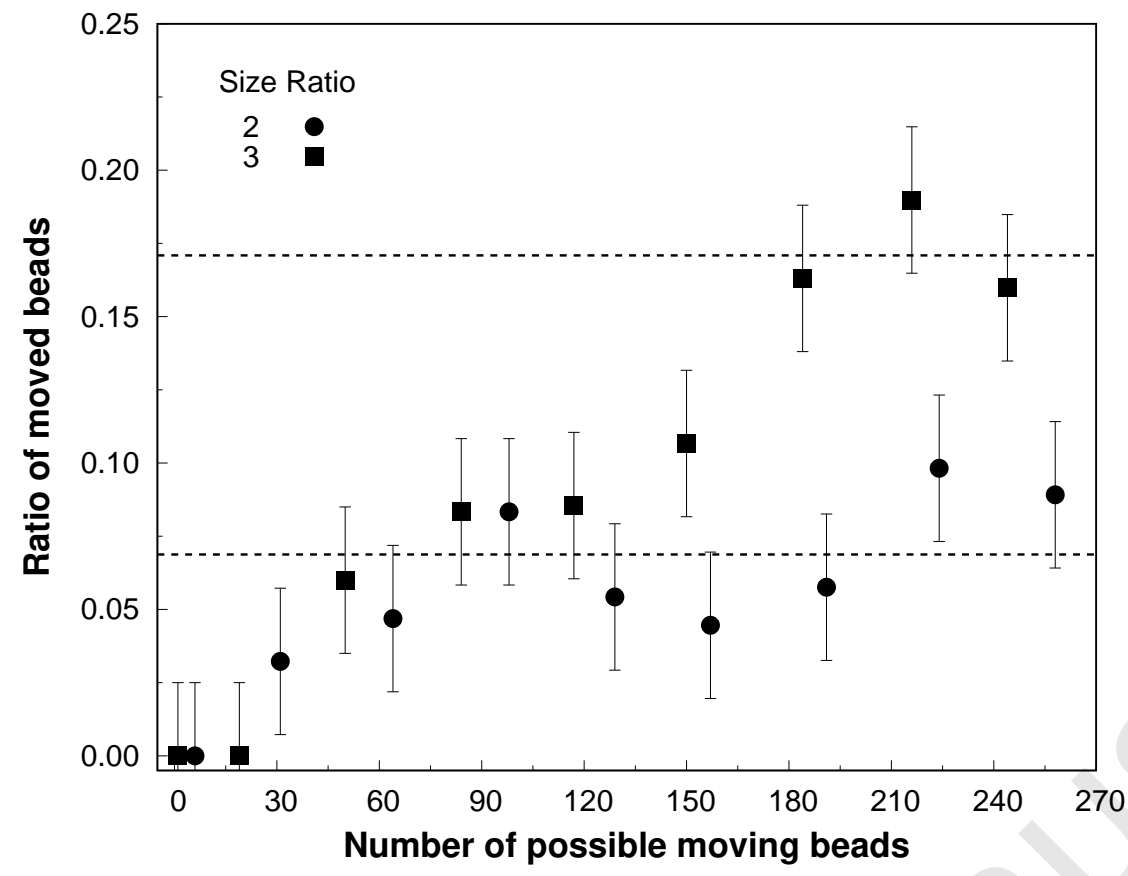

Fig. 13 Evolution of the statistics versus the number of moving beads in the numerical simulations. In this case the frequency is $10 \mathrm{~Hz}$ and the nondimensional amplitude is 2 . The bars indicate the errors.

coefficient going from 0 to 0.3 is found. Consequently, the selected values for all the particles (mobile and glued) are $10^{6} k N$ for the stiffness, 0.1 for the rolling resistance coefficient, and 0.3 for the friction coefficient. On the other hand, humidity percentages are important and the values at the laboratory during the experiments are taken into account. Thus, relative humidity is fixed to a value close to $50 \%$. Table 1 presents all the physical parameters used by default or tried in the present simulations. For a high coverage value, the simulation for one amplituderatio-frequency value takes one week using a processor on PowerEdge $R-930$ for 16.000.000 time steps, which corresponds to the order of 16 seconds in real time. 


\begin{tabular}{|c|c|c|}
\hline Parameters & Default value & Range of values tested \\
\hline Diameter of glued spheres $D$ & 1 or $2 \mathrm{~mm}$ & 1 or $2 \mathrm{~mm}$ \\
\hline Density of glued spheres & $2500 \mathrm{~kg} \cdot \mathrm{m}^{-3}$ & $2500 \mathrm{~kg} \cdot \mathrm{m}^{-3}$ \\
\hline surface dimension & $60 \times 60 \mathrm{D}$ & $60 \times 60 \mathrm{D}$ \\
\hline Percentage of covering rate & $35 \%$ to $82 \%$ & $35 \%$ to $82 \%$ \\
\hline & $(4500$ to 10500$)$ & \\
\hline Diameter of moving spheres & 1 to $7 \mathrm{~mm}$ & 1 to $7 \mathrm{~mm}$ \\
\hline Percentage of moving spheres & $6 \%$ of glued spheres & 30 to 800 by number \\
\hline Density of moving spheres & $2500 \mathrm{~kg} \cdot \mathrm{m}^{-3}$ & 1000 to $8000 \mathrm{~kg} \cdot \mathrm{m}^{-3}$ \\
\hline Restitution coefficient ( $b_{N}$ linked to it) & 0.6 & 0.4 to 0.8 \\
\hline Friction coefficient $\mu$ & 0.5 & 0.3 to 0.5 \\
\hline Tangential friction coefficient $b_{t}$ & $0.3 b_{N}$ & $0.3 b_{N}$ \\
\hline Stiffness $k_{n}$ & $1.10^{6} \mathrm{kN}$ & $1.10^{5} \mathrm{kN}$ to $1.10^{8} \mathrm{kN}$ \\
\hline Tangential Stiffness $k_{t}$ & 0.3 & 0 to 0.3 \\
\hline humidity rate & $50 \%$ & 0 to $70 \%$ \\
\hline Rolling resistance coefficient & 0.1 & 0 to 0.3 \\
\hline Frequency oscillation & 3 to $25 \mathrm{~Hz}$ & 3 to $25 \mathrm{~Hz}$ \\
\hline Frequency amplitude & 1 to $5 \mathrm{D}$ & 1 to $5 \mathrm{D}$ \\
\hline Number of time-steps & $1.610^{7}$ & $1.610^{7}$ to $1.610^{9}$ \\
\hline
\end{tabular}

Table 1 Physical parameters used in the present simulations.

\subsection{Analysis of the results}

Once the mechanical conditions are defined for the simulations, we are able to examine the influence of key parameters such as the coverage of the surface, the size ratio between the mobile and glued particles and the amplitude of the oscillation.

Figure 14 shows the comparison between simulation results and experiments for $S 1$. The vertical series of filled symbols defined for two different amplitudes in each figure correspond to the different size ratios used in our numerical model, i.e., 

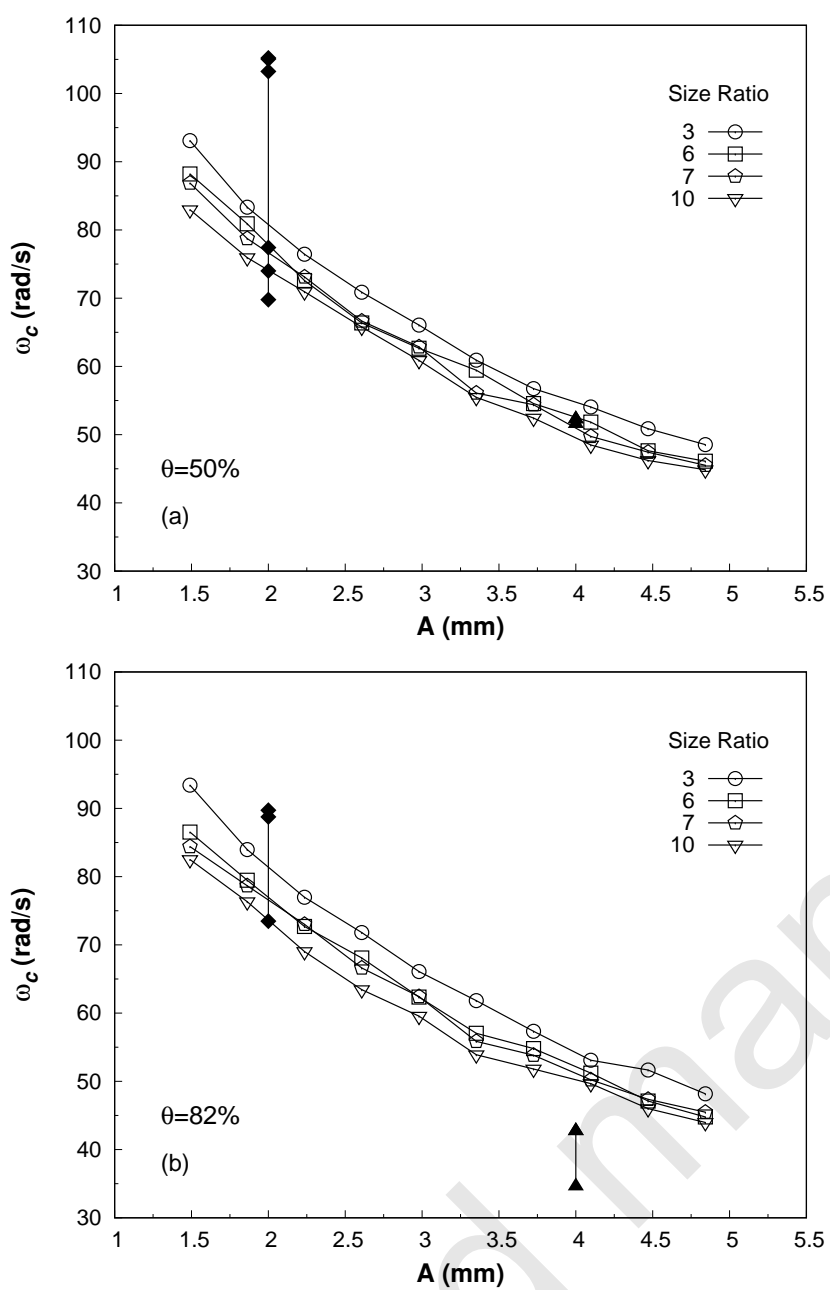

Fig. 14 Comparison for $\omega_{c}$ versus the amplitude of the oscillation, the size ratio and the coverage for $S 1$. (a) $\theta=50 \%$ (a) and $\theta=82 \%$ (b).

the size of the moving spheres compared to the size of the glued ones. In part (a), the symbols from top to bottom at $A=2 \mathrm{~mm}$ are equal to $1.5,2,3,5,6,7$ and, at $A=4 m m$, they are 1.5, 2 and 3 , respectively, and are practically overlapped. In part (b), the ratios correspond to 1.5, 2 and 3 for the two values of the amplitude. Recall here that the low ratio limit cannot be reached in experiments due to the 
difficulty for detecting the movement of particles when their size is close to that of the glued spheres on the surface.

For the case of small amplitudes and low ratios, the frequencies practically overlap, i.e., the particles are trapped inside local holes, especially for the lower coverage. On the other hand, at small amplitudes, the spread out of the frequency values is larger than for larger amplitudes. This is especially evident in Fig. 14(a), where the range of numerical and experimental values at $A=2 \mathrm{~mm}$ is quite larger than that for $A=4 \mathrm{~mm}$. As said before, the effect of the size ratio is more important at lower amplitudes, where the particles get trapped.

It is worthy to mention that in Fig. 14(b), a good coincidence is found at low amplitude but, for the case of high amplitude, simulations overvalue the experimental results. We attribute this difference to the fact that the possible positions of the mobile spheres in the experiments can be biased by the local inhomogeneity made during the construction of the surface, especially when we are approaching the maximal random close packing $(82 \%)$.

A series of tests were also performed to check whether the density of the moving particles modifies the critical frequencies or not. In the experiments, although the decaying behavior of $w_{c}$ vs. $A$ is different for glass than for ABS (see figures 4 to 7 ), the critical values do not differ too much from one case to the other. In the simulations, we verify that this is also the case, even for different coverage values. Figure 15 shows the behavior found for materials whose density is higher and lower than that of glass. As seen, density is not a crucial parameter in these experiments.

To check a wider range of coverage compared to that used in experiments, simulations are performed to obtain the critical frequencies as a function of $\theta$ for 


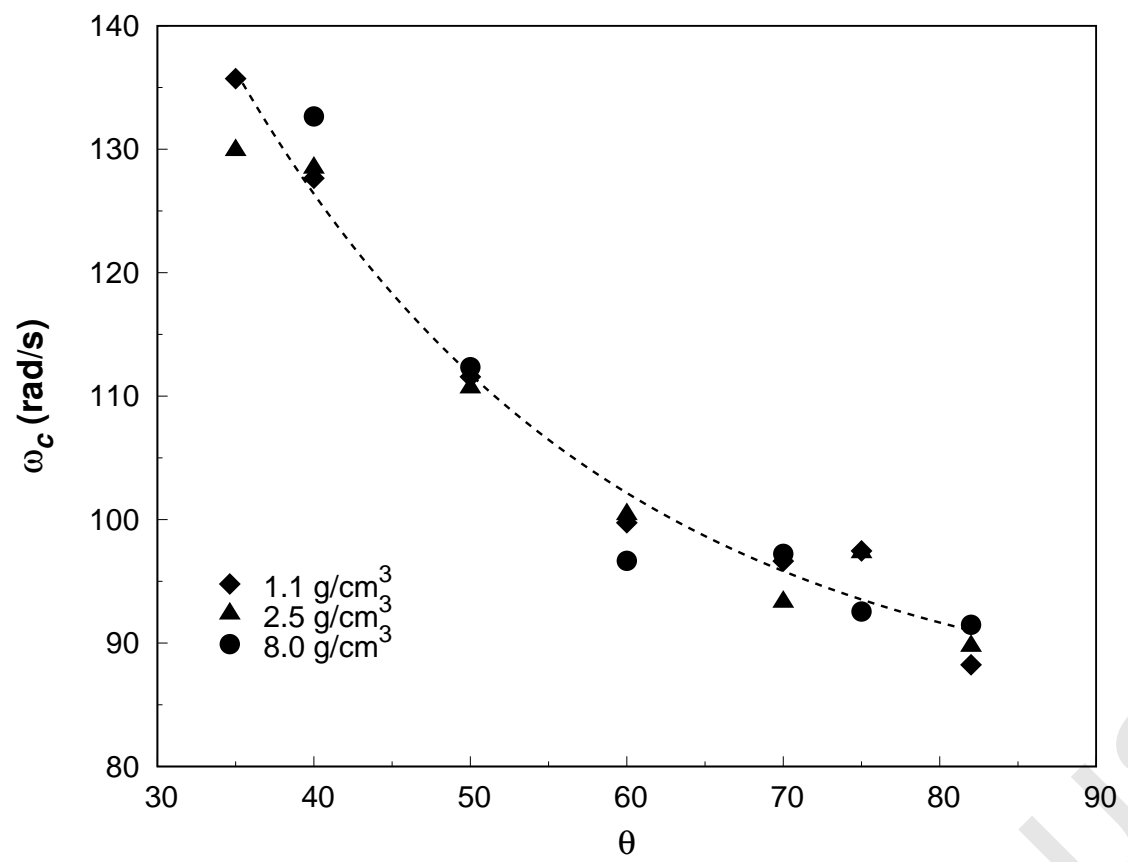

Fig. 15 Evolution of the threshold for three different densities for moving beads with a diameter of 1.5 times the diameter of the fixed beads and an amplitude of $1 \mathrm{~mm}$.

three different values of $A$ (see Fig. 16). Besides the fact already proven in experiments that $w_{c}$ decreases with $A$, the coverage incidence is more important when $\theta$ is lower than $60 \%$ at all amplitude values. This explains why in our experiments, the role of the coverage is weak.

Finally, Fig. 17 shows the role played by the coverage in the critical frequency values at a low amplitude. As expected, the larger $\theta$ is, the lower is $w_{c}$, i.e., local holes are smaller. Nevertheless, and for the range of coverage used in the experiments, the moderate role of $\theta$ is corroborated through the simulations, given that from $\theta=60 \%$ up, the threshold is quite the same. On the other hand, the numerical results evidence that the size ratio is especially important for high values of $\theta$. Indeed, this feature is also observed in the present experiments. For instance, 


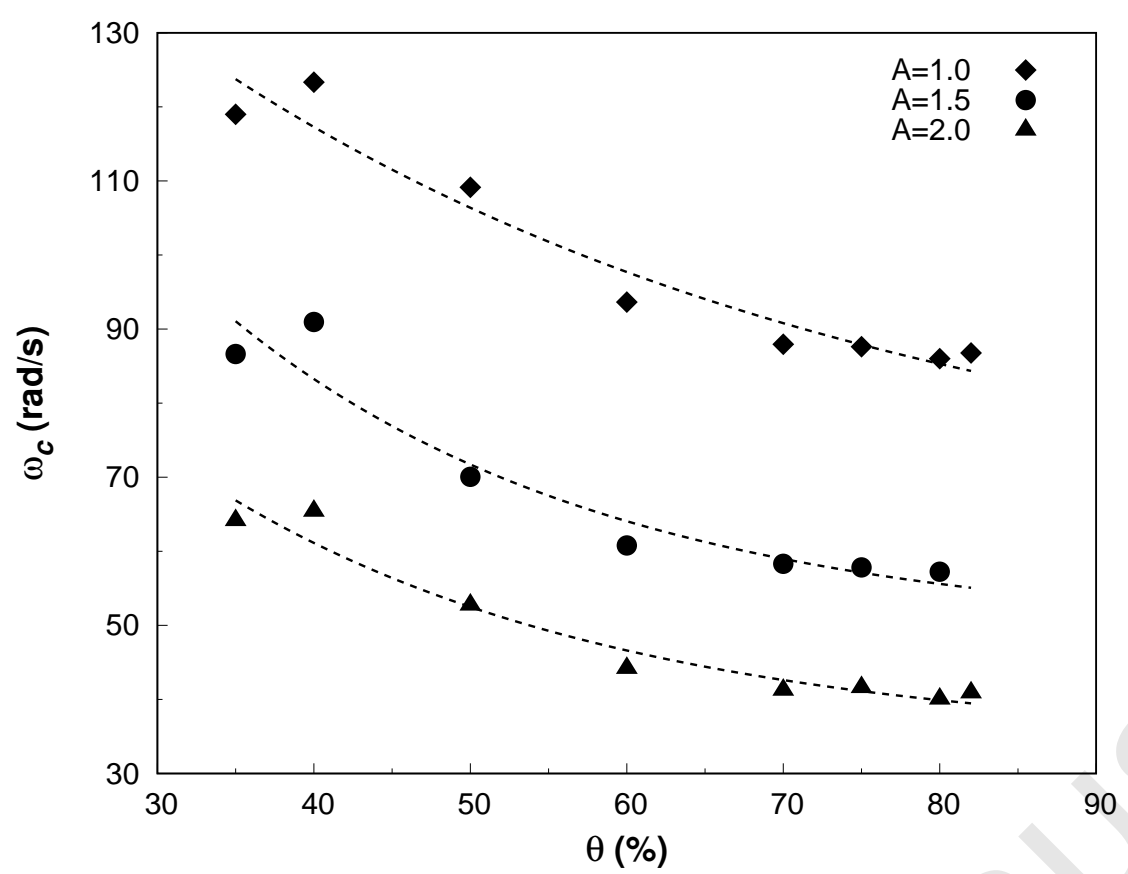

Fig. 16 Evolution of the threshold versus the amplitude of the oscillations for moving beads with a size ratio equal to 1.5 .

in Fig. 3, the difference between the curves for $S 1$ and $S 2$ is greater than for $\theta=50 \%$.

\section{Conclusions}

The experimental results shown so far, and the simulations performed over a larger range of values for coverage and size ratios, show a good agreement. They allow to describe the problem of the movement initiation of millimeter particles on rough surfaces subjected to vertical vibration and the following conclusions can be drawn.

The critical frequencies needed to destabilize the oscillating particles follow an exponential decay with the amplitude of the oscillation. 


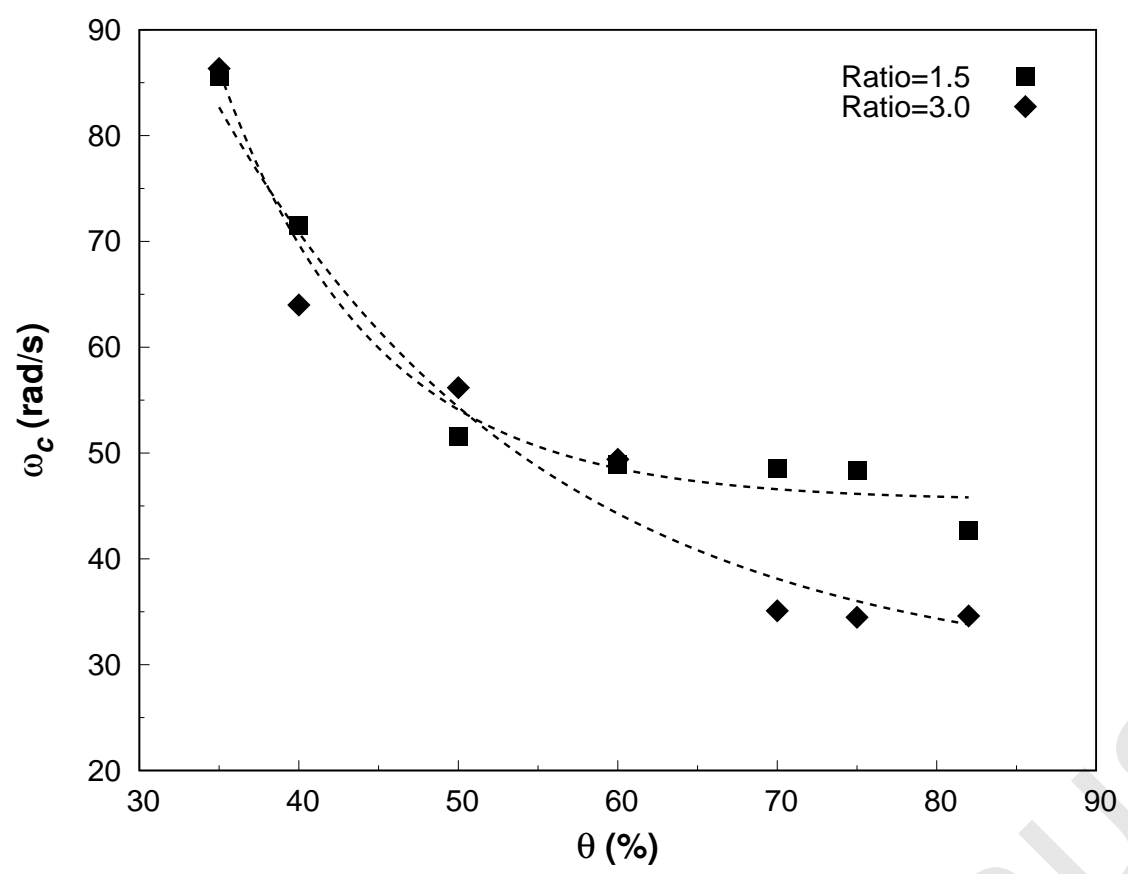

Fig. 17 Evolution of the threshold for two different size ratio between moving beads and fixed ones for an amplitude of $2 \mathrm{~mm}$.

Considering two parameters in the exponential fitting of the data, i.e., the preexponential factor $\omega_{c}(0)$ and the decaying constant $\gamma$, we find that, as the size of the particle is greater, $\omega_{c}(0)$ is lower and $\gamma$ keeps practically constant. These results also lead to conclude that the torque needed to destabilize the particles decreases as the size ratio between moving and glued particles increases.

A weak dependence on $\theta$ is found in the experiments, while simulations prove that it begins to be important when $\theta \leq 60 \%$.

On the other hand, the experimental results demonstrate that density is not a crucial parameter here, and this feature is also reinforced by the simulations.

In summary, in the process of destabilization of millimeter particles on vibrated rough surfaces with a relatively high coverage, the size of the mobile particle plays 
the main role in the determination of the frequency-amplitude pairs for movement initiation.

Acknowledgements This works is partially supported by the LIA Physique et Mécanique des Fluides and the ECOS-SUD A15E03. Authors also acknowledge the financial support from CONICET and Universidad Nacional de San Luis through grants 353 and 03-2718.

\section{Compliance with ethical standards}

\section{Conflict of interest}

The authors declare that there is no conflict of interests regarding the publication of this article.

\section{References}

1. G.L. Dybwad, J. Appl. Phys. 58, 2789 (1985)

2. G. Ziskind, M. Fichman, C. Gutfinger, J. Aerosol Sci. 31, 703 (2000)

3. W. Theerachaisupakij, S. Matsusaka, M. Kataoka, H. Masuda, Advanced Powder Technology 13(3), 287 (2002). DOI 10.1163/156855202320252453

4. Y. Mawatari, T.K.Y. Tatemoto, S. Uchida, K. Noda, Powder Technol. 123, 69 (2002)

5. N. Tippayawong, I. Preechawuttipong, Australian Journal of Basic and Applied Sciences 5, $356(2011)$

6. G. D’Anna, G. Gremaud, Physical Review E 64(1), 011306 (2001). DOI $10.1103 /$ physreve.64.011306

7. P. Philippe, D. Bideau, Europhysics Letters 60, 677 (2002)

8. S. Herminghaus, Advances in Physics 54(3), 221 (2005)

9. P. Richard, M. Nicodem, R. Delannay, P. Ribière, D. Bideau, Nature Materials 4, 121 (2005). DOI doi:10.1038/nmat1300

10. I.Sañchez, F. Raynaud, J. Lanuza, B. Andreotti, E.C. ement, I.S. Aranson, Physical Review E 76, 060301(R) (2007) 
11. K. Hein, T. Hucke, M. Stintz, S. Ripperger, Part. Part. Syst. Char. 19, 269 (2002)

12. F. Soepyan, S. Cremaschi, B. McLaury, C. Sarica, H. Subramani, G. Kouba, H. Gao, Powder Technol 292, 272 (2016)

13. K.A.V. Aracena, J.G. Benito, L. Oger, I. Ippolito, R.O. Uñac, A.M. Vidales, Particuology 40, 1 (2018). DOI 10.1016/j.partic.2017.11.009

14. S.B. Savage, in Disorder and Granular Media, ed. by D. Bideau (North Holland, Amsterdam, 1993), pp. 255-287

15. L. Oger, I. Ippolito, A. Vidales, Granul Matter 9, 267 (2007)

16. E. Charlaix, J. Crassous, J. Chem. Phys. 122, 184701 (2005)

17. J. Crassous, M. Ciccotti, E. Charlaix, Langmuir 27(7), 3468 (2011)

18. J. Ai, J.F. Chen, J.M. Rotter, J.Y. Ooi, Powder Technology 206(3), 269 (2011). DOI 10.1016/j.powtec.2010.09.030. URL http://dx.doi.org/10.1016/j.powtec.2010.09.030

19. J. Huang, M.V. da Silva, K. Krabbenhoft, Computers and Geotechnics 49, 289 (2013). DOI 10.1016/j.compgeo.2012.08.007

20. C. Zhaoa, C. Li, Physica A 460, 44 (2016). DOI 10.1016/j.physa.2016.04.043

21. S. Torquato, Phys. Rev. E 51, 3170 (1995) 